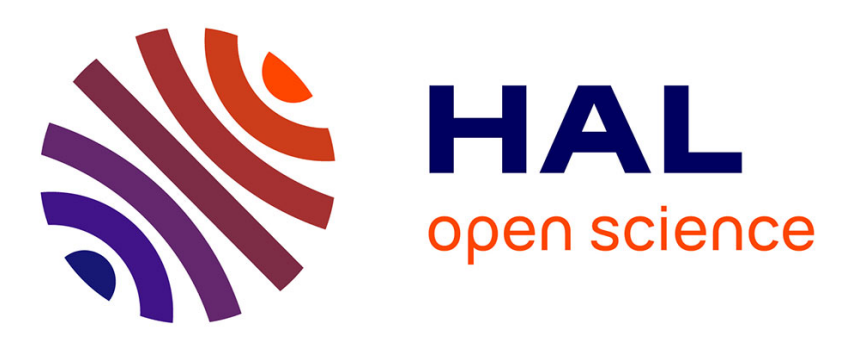

\title{
Orientations, caractères et composition des migrations Sud-Sud
}

\author{
Sylvain Souchaud
}

\section{To cite this version:}

Sylvain Souchaud. Orientations, caractères et composition des migrations Sud-Sud. Christophe Jaffrelot; Christian Lequesne. L'enjeu mondial. Les migrations, Presses de Sciences Po, pp.107-114, 2009. halshs-00553015

\section{HAL Id: halshs-00553015 https://shs.hal.science/halshs-00553015}

Submitted on 26 Jan 2011

HAL is a multi-disciplinary open access archive for the deposit and dissemination of scientific research documents, whether they are published or not. The documents may come from teaching and research institutions in France or abroad, or from public or private research centers.
L'archive ouverte pluridisciplinaire HAL, est destinée au dépôt et à la diffusion de documents scientifiques de niveau recherche, publiés ou non, émanant des établissements d'enseignement et de recherche français ou étrangers, des laboratoires publics ou privés. 


\title{
Orientations, caractères et composition des migrations Sud-Sud
}

\author{
Sylvain Souchaud
}

Sylvain Souchaud est géographe, chargé de recherche à l'Institut de recherche pour le développement (IRD) et au Núcleo de estudos de população (NEPO) à l'universidade estadual de Campinas (Unicamp) dans l'Etat de São Paulo. Il travaille sur les transformations spatiales liées aux mouvements de populations, internes et internationaux, aux frontières internationales et, plus récemment, dans les métropoles.

En 2005, les Nations unies estimaient à 191 millions d'individus le volume mondial de migrants internationaux (tableau 1), soit environ $3 \%$ de la population de la planète. La population migrante a augmenté de façon continue et soutenue depuis près de cinquante ans, puisqu'elle a été multipliée par 2,5 entre 1960 et 2005. Considérée à l'aune de la population totale mondiale, la population de migrants a cru sur l'ensemble de la période, passant de $2,1 \%$ et $3 \%$ de la population mondiale.

Les migrations Sud-Sud représentent une part importante du total des échanges migratoires. Il est difficile de mesurer précisément les flux qui les composent. Jusqu'à la fin des années 1980, les mouvements de population en direction du Sud étaient les plus nombreux. Mais, aujourd'hui, les pays du Sud fixent moins de $40 \%$ du total des mouvements migratoires de la planète, soit environ 75 millions d'individus. En revanche, ils sont à l'origine des deux tiers au moins de l'émigration mondiale des deux dernières décennies.

Trois phénomènes - la transition démographique, intervenant dans l'ensemble des pays du Sud mais à des stades d'avancement divers, la mondialisation, favorisant l'intégration tout autant que la différenciation des espaces, et enfin la redéfinition des équilibres géopolitiques à l'échelle mondiale - modifient en profondeur les modèles migratoires et les profils des migrants internationaux. Comment s'organisent les flux migratoires du Sud? Comment se distribuent-ils dans l'espace? Quelles sont leurs spécificités ? Leur nature et leur composition leur confèrent-elles une spécificité dans l'ensemble des mouvements migratoires internationaux ?

\section{Mesure et orientations des flux}

La mesure statistique des échanges migratoires entre régions continentales et entre Sud et Sud n'est pas chose aisée. Certaines difficultés tiennent aux sources. La qualité des recensements est globalement imparfaite. C'est un fait connu, mais il s'applique fortement aux données relatives aux migrants, population dont la part en situation irrégulière, estimée par les Nations unies à $15 \%-20 \%$ du total, est probablement sous-enregistrée dans les recensements nationaux.

Par ailleurs, les données statistiques sont construites à partir de l'information recueillie dans les pays d'accueil, si bien que c'est l'immigration et non l'émigration (en tout cas pas systématiquement au point que l'on puisse avoir une vue globale du phénomène) qui est renseignée. Cette dernière doit être décrite à partir de la compilation des données censitaires de l'ensemble des lieux de résidence, c'est-à-dire pays par pays. Dès lors, établir des matrices 
migratoires à l'échelle globale est fastidieux. Les organismes internationaux travaillant sur la question, la Division de la population des Nations unies (UNPD) en tête, ne résolvent qu'en partie le problème car, soit les données livrées sont limitées à un espace géographique, soit elles concernent l'ensemble du globe mais ne prennent en compte que les flux entrants. Dans tous les cas, il est très difficile d'établir et de mesurer la dispersion sur la surface de la terre d'une population émigrée.

Enfin, les statistiques captent, à partir d'un événement migratoire passé (généralement le départ du pays de naissance), des stocks de migrants, c'est-à-dire des individus sédentarisés et non à proprement parler des populations en mouvement.

Nous manipulons ainsi des estimations statistiques qui observent le phénomène migratoire selon des choix précis et en fonction de contraintes particulières et qui aboutissent à un déséquilibre de l'état des connaissances entre immigration et émigration. Associer immigration et émigration dans une même approche n'étant possible que sur certains flux, nous serons contraints de les dissocier à l'échelle globale. Ces précisions apportées, quelles observations peut-on faire des mouvements migratoires entre pays du Sud au début du XXI siècle?

\section{Distribution des migrations internationales au Sud}

Si l'on reproduit le découpage géographique des Nations unies (UNPD, 2006), les pays du Sud correspondant aux pays en voie de développement ("less developped countries»), regroupent l'Asie, à l'exception du Japon, l'Afrique, l'Amérique latine et les Caraïbes, la Mélanésie, la Micronésie et la Polynésie. En 2005, cette aire géographique, concentrant $81,3 \%$ de la population mondiale, recevait $39,5 \%$ du total des migrants internationaux (qui sont principalement, mais pas seulement, originaires du Sud). L'année 2005 enregistrait donc la confirmation de l'inversion d'une tendance historique, intervenue à la fin des années 1980, suivant laquelle les pays du Sud cessent peu à peu d'être les principaux pays de destination des migrants internationaux. Cette perte d'attractivité s'explique en partie par l'évolution de la population des réfugiés, très majoritairement installée dans les pays du Sud, dont le volume décroît depuis le début des années 1990 ; ils étaient ainsi 18,5 millions en 1990 concentrés à $89,2 \%$ dans les pays du Sud et 9,9 millions en 2006 seulement.

Avec 53,3 millions d'immigrants en 2005, l'Asie est, après l'Europe, le deuxième continent d'accueil de l'ensemble des migrants internationaux (graphique 1). Quant à l'Afrique, elle arrive en quatrième position, après l'Amérique du Nord. Mais si l'on observe le poids des immigrants internationaux dans la population continentale, il apparaît que les immigrants d'Asie, d'Afrique et d'Amérique latine et Caraïbes ne représentent pas $2 \%$ de la population, alors qu'ils constituent entre $8 \%$ et $15 \%$ de la population totale en Europe, en Amérique et en Océanie (graphique 2).

L'Asie (sans le Japon) polarise 68,1\% des flux mondiaux de migrants internationaux installés aux Sud, l'Afrique $22,7 \%$ et l'Amérique latine et les Caraïbes 8,8\%. Cette répartition correspond grosso modo aux équilibres démographiques généraux au Sud, où l'Asie (sans le Japon) pèse pour $71,9 \%$ de la population totale, l'Afrique $17,3 \%$ et l'Amérique du Sud $10,7 \%$.

Cette analyse des migrations au Sud est peu pertinente à ce niveau de généralité. En revanche, si l'on observe la distribution migratoire par pays d'accueil (UNPD, 2006), des effets de lieux apparaissent : certains pays polarisent les mouvements de populations, quand d'autres, parfois contre toute attente, semblent être en marge des routes migratoires. On sait qu'à l'échelle globale $12 \%$ des pays regroupent $75 \%$ des migrants. Dans les pays du Sud, les migrants s'orientent vers un nombre limité de destinations et la concentration peut être absolue, c'est-à- 
dire correspondre à l'installation d'une population nombreuse de migrants dans un même pays (300 000 Brésiliens vivent au Paraguay qui compte 6 millions d'habitants) et/ou relative (le même nombre vivent aux Etats-Unis), lorsqu'elle représente un effectif important de la population totale du pays. L'Inde est le premier pays d'accueil, avec 5,7 millions d'immigrants en 2005, ceux-ci ne représentent pourtant que $0,5 \%$ de la population indienne. $\mathrm{Au}$ Kazakhstan, les 2,5 millions d'immigrants comptent pour 16,9\% de la population. Situation identique en Côte d'Ivoire, où 2,4 millions d'immigrants équivalent à $13,1 \%$ de la population. La Chine et le Brésil sont des exemples atypiques. En Chine, l'immigration est à tous égards de faible ampleur, les 0,6 million d'immigrants pesant à peine $0,1 \%$ de la population nationale. Puissance démographique et puissance économique émergente, le pays se trouve apparemment en marge des trajectoires migratoires internationales. Sur le continent américain, la situation du Brésil est quelque peu semblable. En 2005, 641536 émigrants ont été recensés au Brésil (dont environ 150000 seulement sont originaires du Sud) sur une population totale de près 190 millions d'habitants.

$\mathrm{Si}$, en dépit des difficultés méthodologiques évoquées plus haut, nous étudions l'origine des migrants, on constate que certains Etats du Sud sont plus des pays d'émigration que d'immigration. La Chine est le premier pays d'émigration avec 35 millions de personnes dispersées dans de nombreux pays au Nord comme au Sud. Elle est suivie de 1'Inde (20 millions d'émigrés) et les Philippines (7 millions) (Guengant 2006). Les pays africains ne comptent pas parmi les principaux pays d'émigration.

Dans certains pays, l'émigration s'apparente, par son importance, à une véritable hémorragie humaine. L'intensité migratoire se définit par rapport à une situation sociale, économique ou politique, conjoncturelle ou structurelle, limitant l'accès aux ressources essentielles de contingents croissants de populations fragilisées. Le départ d'une partie des jeunes actifs, très qualifiés (brain drain) ou sans qualification, déstabilise davantage les systèmes politiques, économiques, sociaux (les structures familiales notamment) fragiles, sans que le bénéfice des effets compensatoires, à travers les remittances, soit avéré sur le long terme. L'émigration paraguayenne, principalement à destination de l'Argentine, ou l'émigration nicaraguayenne au Costa Rica en sont des illustrations. Les effectifs pourront sembler modestes, 323000 immigrants paraguayens recensés en Argentine en 2000 et 226000 immigrants nicaraguayens recensés au Costa Rica la même année, mais l'Amérique latine est un souscontinent de faibles densités rassemblant des pays de faible poids démographique $(6,3$ millions d'habitants au Paraguay, 5,6 millions au Nicaragua) pour lesquels ces départs sont lourds de conséquences.

Une part importante des mouvements migratoires se déploient sur de courtes distances. La migration s'organise entre pays voisins, et pas uniquement lorsqu'il s'agit d'une migration d'urgence comme ce fut fréquemment le cas en Afrique ou en Amérique centrale ces dernières décennies avec les mouvements des réfugiés. La proximité géographique qui caractérise le mouvement migratoire s'accompagne d'une proximité historico-culturelle, essentielle. Les systèmes migratoires régionaux s'articulent autour d'un pôle, dont la fonction de moteur économique est souvent l'héritage d'un système colonial et de sa dynamique d'organisation des espaces (Amin 1973). Il en est ainsi de la centralité ivoirienne en Afrique de l'Ouest, initiée ou relancée par la promotion coloniale des cultures d'exportation qui convertirent ce pays en "eldorado » pour de nombreuses populations originaires des pays frontaliers (Burkina Faso et Ghana). Dans la partie méridionale du continent, l'Afrique du Sud voit converger les routes migratoires de la main-d'oeuvre de plusieurs pays voisins, en provenance notamment du Zimbabwe ou du Mozambique. Certes le Mozambique ne possède pas le même passé colonial que l'Afrique du Sud, mais la longue guerre civile (1976-1992) qui s'est déroulée dans ce pays a montré l'étroitesse des liens ambigus et conflictuels qui unissent ces deux pays. En Amérique latine, on peut citer l'Argentine, où les migrants 
paraguayens et boliviens affluent depuis environ cinquante ans ; en Asie, la Malaisie, pôle migratoire régional dont le dynamisme initial repose sur l'économie de plantation.

\section{Types migratoires et profils des migrants}

Les moteurs de la migration dépendent des évolutions politiques, économiques et culturelles qui s'inscrivent dans la mondialisation, compris comme un mouvement de développement et d'intégration généralisé à l'ensemble de la planète construit autour d'un modèle culturel de référence, mais facteur de forts déséquilibres socio-spatiaux.

Il n'existe pas véritablement de causes spécifiques aux migrations Sud-Sud qui les distingueraient des migrations Sud-Nord, tout au plus observe-t-on certaines différences, plus affirmées.

Ainsi, les mouvements de réfugiés, en dépit d'une diminution notable de cette population, demeurent nettement marqués par le cadre géographique du Sud. En 2005, les réfugiés représentent $7,7 \%$ du total mondial des migrants internationaux. Cette proportion atteint $14,4 \%$ des migrants installés au Sud et même 23,2 \% du total des immigrants présents dans les cinquante pays les moins avancés, mais seulement $2,3 \%$ des immigrants dans les pays développés. Si les réfugiés, entendus au sens strict de la convention de Genève (1951), sont en diminution, les migrations forcées liées à des bouleversements environnementaux sont quant à elles en augmentation. Les réfugiés environnementaux ont été identifiés pour la première fois au début des années 1980 ; à la suite de l'accélération des changements climatiques, leur nombre pourrait atteindre environ 200 millions d'ici à 2050 (Domenach, 2008). Nul doute qu'une partie importante d'entre eux traverseront des frontières internationales. Les pays du Sud, plus vulnérables, seraient plus sévèrement affectés, Songeons par exemple aux effets en termes de peuplement de la montée des eaux océaniques dans les grands deltas asiatiques, et en particulier au Bangladesh dont les possibilités de redéploiement de sa population sur le territoire national sont limitées.

Les migrations de transit représentent également un volume important et croissant des migrations entre pays du Sud. Elles sont en grande partie la conséquence du durcissement des règles d'admission dans les pays du Nord, de l'augmentation des procédures de contrôle aux frontières et des expulsions des migrants illégaux. Une ceinture migratoire de dessine sur les marges méridionales des pays développés : dans les pays d'Afrique du Nord et en Libye (Le Houérou 2007 ; Bredeloup \& Pliez 2005), dans les pays de l'Est du pourtour méditerranéen (Turquie, Liban), ou encore au Mexique se pressent des migrants, originaires d'Afrique subsaharienne dans le premier cas, du Moyen-Orient dans le deuxième et d'Amérique latine dans le troisième, qui préparent leur entrée dans les «forteresses» du Nord. Bien souvent, ces migrations «intermédiaires» se prolongent dans le temps et l'étape se transforme en destination finale.

Au sein des pays du Sud s'organisent également des flux spécifiques de migrations de maind'œuvre (OIM, 2005). Dans les pays pétroliers du golfe Persique, la manne pétrolière a dans un premier temps favorisé le recours aux populations migrantes des pays proches (Yémen, Soudan, Egypte). A mesure que les activités offertes par contrat aux migrants se diversifient (notamment dans le secteur des services), on fait appel à des personnes venues de pays plus éloignés, du Pakistan, de l'Inde, du Bangladesh, d'Asie du Sud-Est (Corée, Thaïlande, Philippines, Sri Lanka, etc.). Dans les Etats pétroliers, les migrants représentent de $25 \%$ à $70 \%$ de la population totale.

En Amérique latine, les flux internes de main-d'œuvre sont également en augmentation. Si une grande majorité de migrants est peu qualifiée, une part non négligeable possède une formation supérieure. Contrairement à ce qui se passe en Asie, les migrations de main- 
d'œuvre faiblement qualifiée ne sont pas encadrées par des contrats temporaires; par conséquent, la part des travailleurs clandestins semble plus élevée en Amérique latine qu'en Asie.

Enfin, il est intéressant de noter que de nouveaux flux migratoires Sud-Sud intercontinentaux se développent. C'est le cas en particulier des populations chinoises s'installant en Afrique de l'Ouest. Ces nouvelles migrations témoignent de nouveaux équilibres géoéconomiques. Le profil des migrants évolue également. A mesure que les pays du Sud s'urbanisent, la part des migrants ruraux diminue et, de plus en plus souvent, les migrants sont issus des villes de leur pays d'origine. La participation féminine est également en augmentation. Dans les années 1990, près de 800000 femmes originaires d'Asie ont migré au Moyen Orient, la plupart comme employées domestiques. En 2005, plus $65 \%$ de l'émigration philippine était féminine.

Les migrations dans les pays du Sud ont évolué au cours des dernières décennies. La part des migrants installés dans les pays du Sud dans le total de la population migrante a reculé, mais leur participation dans les courants d'émigration reste majoritaire et progresse depuis deux décennies. Cette augmentation est soutenue par la transition démographique (baisse de la mortalité et chute de la fécondité) engagée à des stades divers dans l'ensemble des pays du Sud et qui, peu ou prou, favorise le maintien pendant plusieurs décennies d'une natalité élevée. Elle est également confortée par l'inversion des dynamiques migratoires dans certains pays du Nord, en Italie, en Espagne ou au Portugal. Bien que le nombre des réfugiés soit en nette diminution, ce phénomène migratoire spécifique s'inscrit encore nettement dans le contexte géographique du Sud. Et il est fort probable que les réfugiés « environnementaux » contribueront à augmenter les flux dans les décennies, voire les années à venir.

Dans les Etats du Nord, la fermeture des frontières aux migrants originaires du Sud réduit peu la pression migratoire et favorise le développement des migrations de transit. Les pratiques migratoires de diversifient et nombre d'entre elles constituent des entreprises à hauts risques. Dans le même temps, des transferts de main-d'œuvre intercontinentaux s'organisent en marge des pays du Nord, à destination de pays émergents ou présentant un avantage spécifique. Les migrants du Sud, de moins en moins ruraux, circulent d'une métropole du Sud à une autre suivant des trajectoires complexes; ils font parfois partie de l' "élite » mais le plus souvent, ils sont pauvres, sans qualification et employés dans les services ou l'industrie. Les migrations Sud-Sud ne sont pas spécifiques au sein de la géodynamique migratoire. Néanmoins, parce qu'elles soulèvent des questions particulières dans des contextes variés, elles offrent une possibilité, parmi d'autres, d'éclairer les équilibres globaux.

Sylvain Souchaud est géographe, chargé de recherche à l'Institut de recherche pour le développement (laboratoire Population-environnement-développement) et au Núcleo de estudos da população (NEPO) à l'Universidade estadual de Campinas (UNICAMP) de l'Etat São Paulo. Il travaille sur les transformations spatiales liées aux mouvements de populations, internes et internationaux, aux frontières internationales et, plus récemment, dans les métropoles. 
Bibliographie

Amin, S.,. Le développement inégal. Essai sur les formations sociales du capitalisme périphérique. Paris, Editions de minuit, 1973.

Celade,. « Migración internacional - International migration », Observatorio Demográfico, n¹, 2006. Bredeloup, S. and Pliez, O.. " Migrations entre les deux rives du Sahara », Autrepart, n³6, 2005, pp. 3-20.

de Haas, H. 2008. 'Migration and development: A theoretical perspective', Working papers - International Migration Institute, Vol 9, n61, 2008.

de Haas, H.,. The myth of invasion. Irregular migration from West Africa to the Maghreb and the European Union. Oxford, International Migration Institute, 2007.

http://www.imi.ox.ac.uk/pdfs/WP9\%20Migration\%20and\%20development\%20theory\%20HdH.pdf Domenach, H.,. « Les grandes tendances démographiques et l'environnement: l'enjeu d'une planète viable $»$, Mondes en développement, $\mathrm{n}^{\circ} 142,2008$, pp. 97-111.

Guengant, J.-P.,. « Migrations internationales: Quels impacts sur le développement », Sciences au Sud, $\mathrm{n}^{\circ} 36,2006$.

Le Houérou, F. et al., " Migrations Sud-Sud », Revue des mondes musulmans et de la Méditerranée, 2007, pp. 119-120.

OIM.,. World Migration 2005: Costs and Benefits of International Migration, Genève, OIM, 2005. United Nations Population Division. 2006, World Migrant Stock: The 2005 Revision Population Database, United Nations, http://esa.un.org/migration/index.asp?panel=1 
Tableau 1. Migrants internationaux et réfugiés dans le monde, de 1960 à 2005.

\begin{tabular}{rrrrr}
\hline & $\begin{array}{c}\text { Estimation du nombre de } \\
\text { migrants internationaux (en } \\
\text { millions) }\end{array}$ & $\begin{array}{c}\text { Estimation du } \\
\text { nombre de réfugiés } \\
\text { (en millions) }\end{array}$ & $\begin{array}{c}\text { \% migrants } \\
\text { internationaux dans la } \\
\text { population totale }\end{array}$ & $\begin{array}{c}\text { \% des réfugiés dans } \\
\text { le total des migrants } \\
\text { internationaux }\end{array}$ \\
\hline 1960 & 75,5 & 2,2 & 2,5 & 2,9 \\
1965 & 78,4 & 3,9 & 2,4 & 4,9 \\
1970 & 81,3 & 3,9 & 2,2 & 4,8 \\
1975 & 86,8 & 4,2 & 2,1 & 4,9 \\
1980 & 99,3 & 9,1 & 2,2 & 9,1 \\
1985 & 111,0 & 13,2 & 2,3 & 11,9 \\
1990 & 154,9 & 18,5 & 2,9 & 11,9 \\
1995 & 165,1 & 18,5 & 2,9 & 11,2 \\
2000 & 176,7 & 15,7 & 2,9 & 8,9 \\
2005 & 190,6 & 13,5 & 3,0 & 7,1 \\
\hline
\end{tabular}

Source: Population Division of the Department of Economic and Social Affairs of the United Nations Secretariat, Trends in Total Migrant Stock: The 2005 Revision http://esa.un.org/migration, Thursday, October 09, 2008. 
Graphique 1. Estimation du nombre de migrants internationaux selon l'aire géographique de résidence, entre 1960 et 2005.

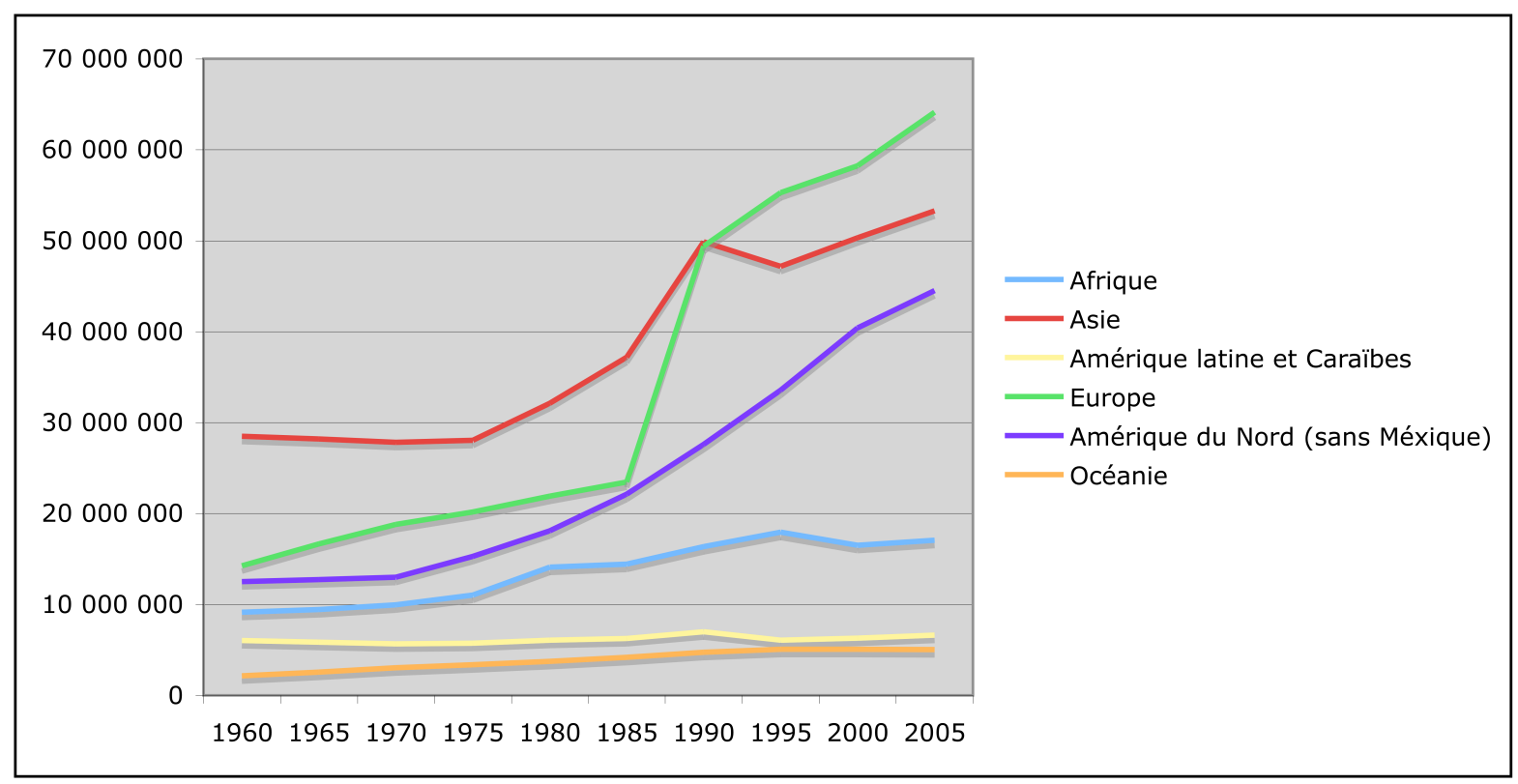

Source: Population Division of the Department of Economic and Social Affairs of the United Nations Secretariat, Trends in Total Migrant Stock: The 2005 Revision http://esa.un.org/migration, Thursday, October 09, 2008. 
Graphique 2. Poids (en \%) des migrants internationaux dans la population totale, selon l'aire géographique, entre 1960 et 2005.



Source: Population Division of the Department of Economic and Social Affairs of the United Nations Secretariat, Trends in Total Migrant Stock: The 2005 Revision http://esa.un.org/migration, Thursday, October 09, 2008. 